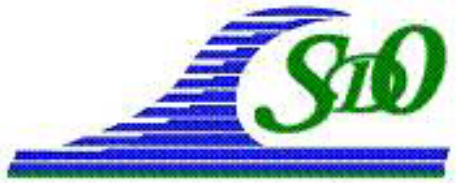

Xìmes Journées Nationales Génie Côtier - Génie Civil

Les Sables d'Olonne, 22-25 juin 2010

DOI:10.5150/jngcgc.2010.007-C C Editions Paralia CFL

disponible en ligne - http://www.paralia.fr - available online

\title{
Modélisation physique des courants d'arrachement : apport des mesures lagrangiennes
}

\author{
Bruno CASTELLE ${ }^{1}$, Hervé MICHALLET ${ }^{2}$, Vincent MARIEU ${ }^{1}$, \\ Fabien LECKLER ${ }^{1}$, Benjamin DUBARDIER ${ }^{1}$, Adrien LAMBERT ${ }^{2,3}$, \\ Céline BERNI $^{2}$, Eric BARTHELEMY ${ }^{2}$, Frédéric BOUCHETTE ${ }^{3}$, \\ Philippe BONNETON ${ }^{1}$, Olivier KIMMOUN ${ }^{4}$, Damien SOUS ${ }^{5}$, Rafael ALMAR ${ }^{1}$ \\ 1. UMR CNRS 5805 EPOC, Université de Bordeaux, Avenue des Facultés, \\ 33405 Talence Cedex, France. \\ b.castelle@epoc.u-bordeaux1.fr \\ 2. Laboratoire des Ecoulements Géophysiques et Industriels (UJF-INPG-CNRS), BP53, \\ 38041 Grenoble, France. \\ 3. Institut de Mathématiques et de Modélisation/Géosciences-M, \\ Université Montpellier 2, Place E. Bataillon, 34095 Montpellier cedex 5, France. \\ 4. UMR 6594, rue Joliot Curie, 13384 Marseille Cedex 13, France. \\ 5. LSEET, Université de Toulon et du Var, 83957 La Garde Cedex, France.
}

\section{Résumé :}

Des mesures lagrangiennes dans des systèmes de courants d'arrachement ont été réalisées au-dessus de 8 morphologies de plage réalistes. Pour chaque morphologie, une grande quantité $(\sim 30)$ d'objets dérivants (drifters) ont été déployés et suivis par un système vidéo. La grande quantité de données acquises a permis de reconstituer la circulation moyenne horizontale et de quantifier pour la première fois la vitesse adimensionnée des courants d'arrachement en fonction du caractère tridimensionnel de la plage. Le taux de rétention des drifters dans la zone de déferlement a été calculé et analysé en fonction de la géométrie et de l'intensité du courant d'arrachement. Ce travail montre que les courants d'arrachement asymétriques (par houle frontale) et les courants d'arrachement transitoires sont ceux qui retiennent le moins les drifters dans la zone de déferlement, ce qui présente un fort intérêt d'un point de vue de la sécurité de la baignade et du mélange des masses d'eau entre le plateau et les franges littorales.

Mots-clés :

Courants d'arrachement - Modélisation physique - Mesures lagrangiennes Circulations horizontales - Courants de baïne - Sécurité de baignade

\section{Introduction}

Les courants d'arrachement (aussi appelés courants sagittaux, courants de baïne sur le littoral aquitain ou rip currents) sont des courants intenses et étroits, dirigés vers le large, qui sont très largement observés le long des plages sableuses dominées par l'action de la houle. Ces courants, qui sont responsables de la majorité des accidents de 
baignade le long des plages, sont associés à des cellules de circulation horizontales responsables d'importants échanges hydro-sédimentaires entre les franges littorales et le plateau continental. Les rétroactions complexes entre ces circulations, les flux sédimentaires et l'évolution du fond sableux (auto-organisation) contrôlent également la dynamique des barres sableuses tridimensionnelles quasi-périodiques de type barres en croissant ou systèmes barre/chenal. C'est pourquoi, les courants d'arrachement ont été très largement étudiés ces dernières années par la communauté internationale (MACMAHAN et al., 2006). Dans cet article nous ne traiterons pas des courants d'arrachement transitoires mais des courants d'arrachement contrôlés par la topographie locale. Ces derniers sont forcés par les gradients de tensions de radiation associées aux vagues au-dessus des plages sableuses tridimensionnelles.

De nombreuses mesures in-situ et en laboratoire de ces courants d'arrachement ont été réalisées cette dernière décennie. En particulier, les mesures sur le terrain ont montré que les courants d'arrachement sont fortement modulés par la marée et les changements de régime de houle (parmi d'autres BRUNEAU et al., 2009). Par conséquent, une grande difficulté des études in-situ des courants d'arrachement est due au fait que l'évolution permanente des régimes de houle et de la hauteur de marée rendent impossible la quantification des courants d'arrachement en fonction de la morphologie $\mathrm{du}$ fond. D'autre part les mesures de courants d'arrachement en laboratoire ont été réalisées au-dessus de morphologies irréalistes (HALLER \& DALRYMPLE, 2001 ; KENNEDY \& THOMAS, 2004). Parmi d'autres limitations de toutes ces expériences, la profondeur relative du chenal était d'un ordre de grandeur supérieur à celle mesurée couramment sur le terrain, ou encore les fortes discontinuités de fond dur généraient le décollement permanent de tourbillons instationnaires pouvant fortement perturber la dynamique du courant d'arrachement et des cellules associées.

Afin de s'affranchir des conditions énergétiques et fortement instationnaires des régimes de houle et de marée sur le terrain, une expérience en laboratoire visant à étudier les courants d'arrachement au-dessus de plages réalistes était nécessaire pour améliorer les connaissances sur la dynamique des courants d'arrachement. Ce besoin a été identifié dans le cadre du projet MODLIT (RELIEFS/INSU) pendant lequel une expérience de 5 semaines pilotée par le LEGI a été réalisée dans le bassin de la SOGREAH au-dessus d'un fond mobile (MICHALLET et al., 2010). Dans cet article, nous décrivons uniquement les résultats obtenus à partir des données lagrangiennes qui ont été réalisées pendant une partie de l'expérience où le caractère tridimensionnel de la plage s'est fortement accentué.

\section{Modèle physique : expérience MODLIT (Bassin LHF)}

Une description plus détaillée de l'expérience sur fond sableux dans le bassin LHF $30 \mathrm{~m}$ x $30 \mathrm{~m}$ est donnée dans MICHALLET et al. (2010). Une schématisation de cette expérience est montrée en figure 1. Ici nous décrirons la dynamique des courants 
d'arrachement pour les 8 morphologies de plages contrastées données en figure 2, pour des conditions de houle constantes, avec une houle frontale de hauteur significative $H s=18 \mathrm{~cm}$ et de période pic $T p=3.5 \mathrm{~s}$.
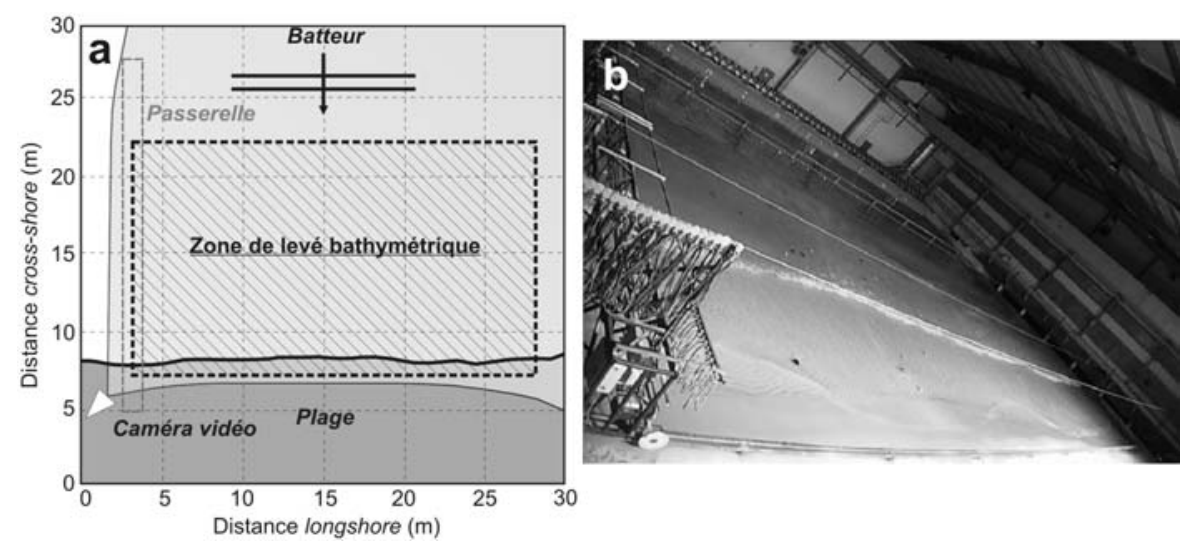

Figure 1. Schématisation de l'expérience avec (a) les dimensions de la plage avec l'emplacement des batteurs et des relevés bathymétriques avec, dans le texte, x la distance longshore et y la distance cross-shore. (b) Iimage de la vidéo montrant la présence des drifters dans la zone de déferlement.

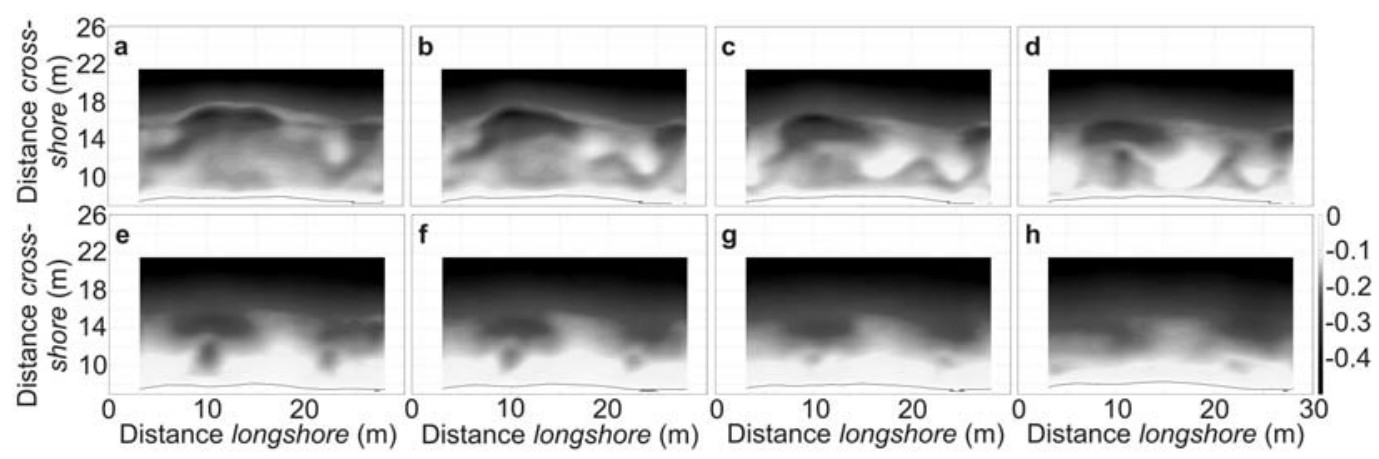

Figure 2. Les 8 configurations pour lesquelles les courants d'arrachement ont été quantifiés. Les caractéristiques de ces morphologies et leur correspondance dans classification de WRIGHT \& SHORT (1984) sont décrites dans le texte.

A noter que, d'une part, ces morphologies sont typiques des systèmes de barres sableuses observées sur le terrain et que, d'autre part, la séquence morphodynamique correspond à une séquence accrétive quasi-complète des états intermédiaires suivant la modélisation conceptuelle de WRIGHT \& SHORT (1984). On observe en effet une morphologie de type Rhythmic Bar and Beach (RBB, figures 2a, 2b et 2c) avec une barre en croissant qui vient se connecter au haut de plage et ainsi former une morphologie de type Transverse Bar an Rip (TBR, figures 2d, 2e et 2f) avec 2 chenaux en $x=11 \mathrm{~m}$ et $x=22 \mathrm{~m}$ (où $x$ est la position longshore). Enfin les chenaux se comblent 
progressivement pour former une morphologie faiblement tridimensionnelle de type Low Tide Terrace (LTT, figures $2 \mathrm{~g}$ et $2 \mathrm{~h}$ ).

Une partie importante de cette expérience a consisté à réaliser des mesures lagrangiennes des circulations induites par les vagues. Pour cela des drifters (ou objets dérivants) devaient être développés. Les drifters devaient flotter pour pouvoir être suivis par la caméra sans subir l'action des vagues (phénomène de surf, c'est-à-dire emportés par le rouleau de déferlement). Après plusieurs essais, la solution adoptée a été de simples ballons de baudruche remplis d'eau. Ceux-ci présentaient l'avantage d'avoir une masse volumique quasi-identique à celle de l'eau, ayant donc le même comportement que les masses d'eau les entourant, tout en présentant une faible surface immergée. Les drifters déployés dans le bassin on été filmés pendant environ une heure pour chaque morphologie. La position de chaque drifter fut ainsi détectée toutes les 6 secondes et transformée de leur position en pixel dans le repère de coordonnées locales liées au bassin. L'évolution de cette position a ensuite été interpolée en temps et en espace afin d'obtenir l'évolution de la vitesse de chaque drifter. Enfin, le domaine du bassin a été divisé en mailles carrées de 1 mètre de côté. Pour chaque maille, les données de vitesse des drifters traversant celle-ci ont été triées et stockées. Pour les mailles comportant plus de 5 données, des valeurs statistiques ont été calculées : courant moyen et écart type en intensité et direction du courant.

\section{Résultats}

\subsection{Circulations moyennes}

La figure 3 montre les circulations moyennes calculées à partir des données lagrangiennes. Les circulations montrent l'omniprésence de courants d'arrachements pour toutes les morphologies avec, visuellement et sans surprise, une intensité des courants d'arrachement qui augmente avec la tridimensionnalité de la plage. On remarque également qu'au début de l'expérience la majorité des données sont acquises dans le courant d'arrachement situé en $\mathrm{x} \approx 10 \mathrm{~m}$ (figures $3 \mathrm{a}, 3 \mathrm{~b}$ et $3 \mathrm{c}$ ) et que progressivement des drifters sont transportés dans l'autre courant d'arrachement en $\mathrm{x} \approx 22 \mathrm{~m}$ (figures $3 \mathrm{~d}, 3 \mathrm{e}, 3 \mathrm{f}, 3 \mathrm{~g}$ et $3 \mathrm{~h}$ ). Par la suite nous focaliserons notre étude sur le courant d'arrachement situé en $\mathrm{x} \approx 10 \mathrm{~m}$ puisque c'est dans celui-ci que le jeu de données acquis est le plus complet.

Une caractéristique importante du courant d'arrachement pendant l'expérience est que celui-ci était parfois symétrique (figures $3 \mathrm{~d}, 3 \mathrm{e}, 3 \mathrm{f}, 3 \mathrm{~g}$ et $3 \mathrm{~h}$ ) et parfois asymétrique (figures $3 \mathrm{a}, \mathrm{b}$ et c), et ce malgré les conditions de houle toujours frontale. Nous verrons par la suite que cela a un impact sur le taux de rétention des courants d'arrachement. Pour la première fois, l'intensité du courant d'arrachement a été quantifiée en fonction de la tridimensionnalité de la plage. Celle-ci a été estimée d'après FEDDERSEN \& GUZA (2003) autour du chenal situé en $x \approx 10 \mathrm{~m}$ par la formule : 


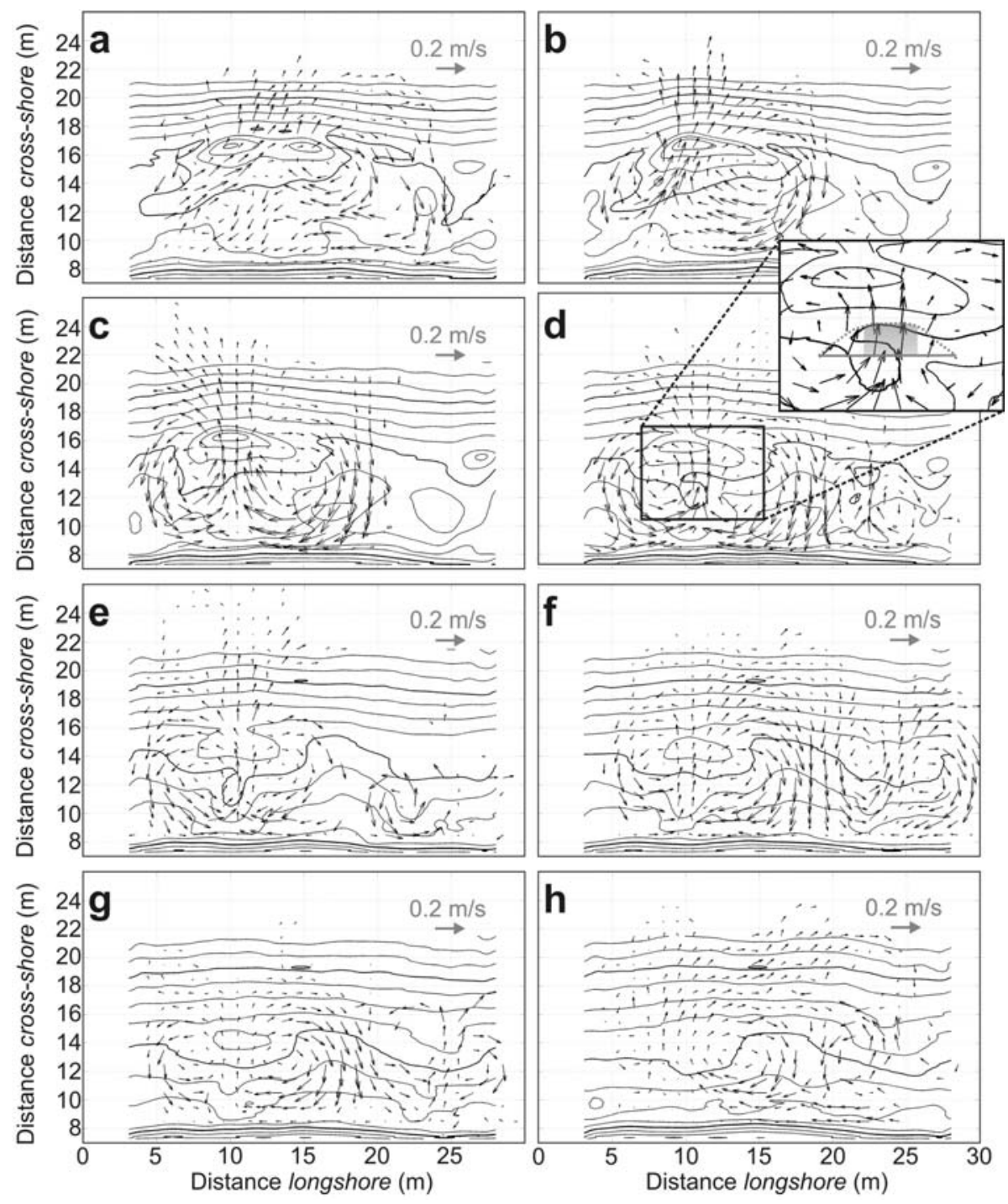

Figure 3. Champs de courants moyens calculés à partir des données lagrangiennes pour les 8 morphologies présentées en figure 2. L'échelle des vitesses est donnée en haut à droite de chaque panel.

$$
\sigma_{\mathrm{h}}(\mathrm{y})=\sqrt{\frac{1}{\mathrm{x}_{2}-\mathrm{x}_{1}} \int_{\mathrm{x}_{1}}^{\mathrm{x}_{2}}\left(\mathrm{~h}(\mathrm{x}, \mathrm{y})-\overline{\mathrm{h}(\mathrm{y}))^{2} \mathrm{dx}}\right.}
$$

où $h(x, y)$ est la cote du fond pour $7.16 \mathrm{~m}<y<22 \mathrm{~m}, x_{l}=3.12 \mathrm{~m}$ et $x_{2}=18 \mathrm{~m}$, et $\overline{h(y)}$ la moyenne longshore entre $x_{1}$ et $x_{2}$. Nous avons ensuite défini la moyenne cross-shore $\overline{\sigma_{h}}$ comme la mesure de la tridimensionnalité du système barre/chenal centré en $x \approx 10 \mathrm{~m}$. Comme aucune définition cohérente de l'intensité du courant d'arrachement $U_{\text {rip }}$ n'est donnée dans la littérature, nous l'avons défini en se basant sur la géométrie des cellules de circulations: $U_{\text {rip }}$ est la moyenne du courant entre les 2 centres des cellules de circulation, le long de la section de $2 \mathrm{~m}$ où il est maximum (figure $3 \mathrm{~d}$ ). Nous 
avons enfin utilisé le nombre de Froude $F r=U_{\text {rip }} /\left[(g h)^{1 / 2}\right]$ comme mesure adimensionnée du courant d'arrachement afin de classifier le régime hydrodynamique. Les résultats sont synthétisés en figure 4 avec et sans prise en compte de la dérive de Stokes. De manière surprenante, une relation presque parfaitement linéaire est obtenue entre l'intensité du courant d'arrachement et la tridimensionnalité de la plage pour une houle donnée.
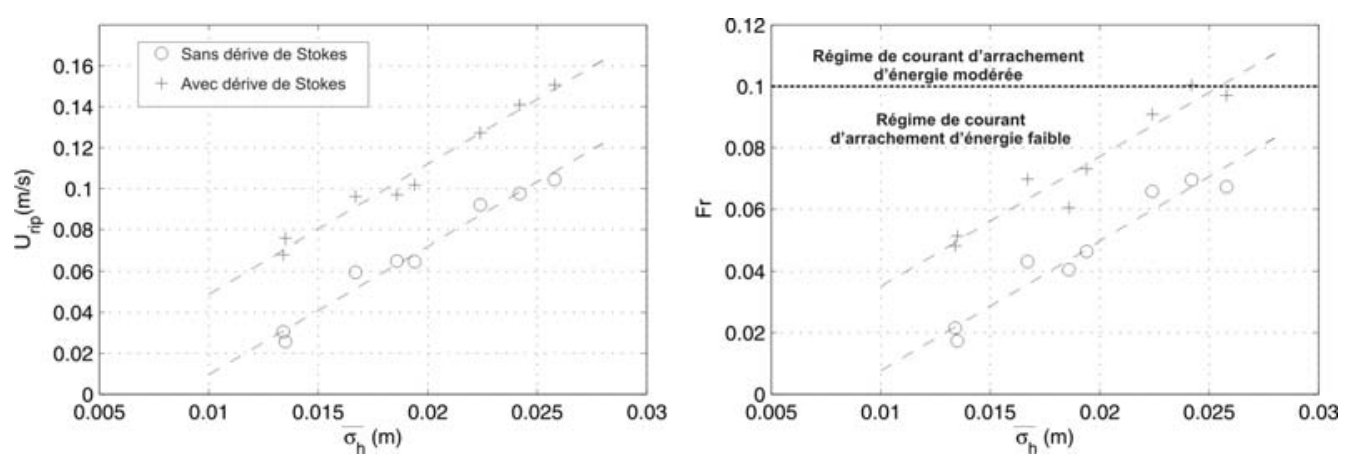

Figure 4. Intensité du courant d'arrachement (a) $U_{\text {rip }}$ et (b) Fr en fonction de la tridimensionnalité de la plage $\overline{\sigma_{z}}$ avec le seuil $F r=0.1$ délimitant les régimes de courant d'arrachement d'énergies faible et modérée selon MACMAHAN et al. (2006).

\subsection{Expulsion des drifters et taux de rétention}

Les circulations moyennes présentées en figure 3 étaient très instables tout au long de l'expérience. Par exemple, comme le montre la figure 5, le courant d'arrachement pouvait passer assez rapidement d'une forme asymétrique (vers la droite ou vers la gauche) à une forme symétrique sans raison apparente. Une autre caractéristique frappante est que les drifters pris dans le courant d'arrachement restaient piégés dans les cellules de circulation avant de rentrer à nouveau dans le courant d'arrachement et ainsi de suite. En de plus rares occasions, les drifters étaient expulsés de la zone de déferlement jusqu'à la zone en face des batteurs à houle (figure 5f). Pour chacune des 8 expériences nous avons compté automatiquement le nombre de drifters entrant dans le courant d'arrachement et ceux expulsés afin de calculer le taux de rétention des cellules de circulation associées au courant d'arrachement. Les résultats sont synthétisés dans le tableau 1. Les résultats montrent que, la plupart du temps, le taux de rétention calculé est de l'ordre de $90 \%$, ce qui est en accord avec les mesures réalisées par MACMAHAN et al. (2010) sur une plage californienne. Toutefois, le taux de rétention chute lorsque le courant d'arrachement moyen est asymétrique (expériences a, b et c). Ces nouveaux résultats sont très importants d'un point de vue de la sécurité de la baignade ou encore des échanges hydro-sédimentaires entre le plateau et les franges littorales. En outre, l'analyse en cours des pulsations du courant d'arrachement n'a pas mis en évidence de lien avec l'expulsion des drifters, contrairement à ce qui est avancé dans la littérature. Enfin, durant l'expérience (h) le taux de rétention est très faible alors que le courant 


\section{XI $I^{\text {èes }}$ Journées Nationales Génie Côtier - Génie Civil}

Les Sables d'Olonne, 22-25 juin 2010

d'arrachement est à la fois faible et symétrique. Nous pouvons expliquer ce faible taux de rétention par l'apparition de courants d'arrachement dits «transitoires », qui, par définition, ne retiennent que très peu les masses d'eau, puisque la bathymétrie autour de $x \approx 10 \mathrm{~m}$ était devenue quasiment homogène.
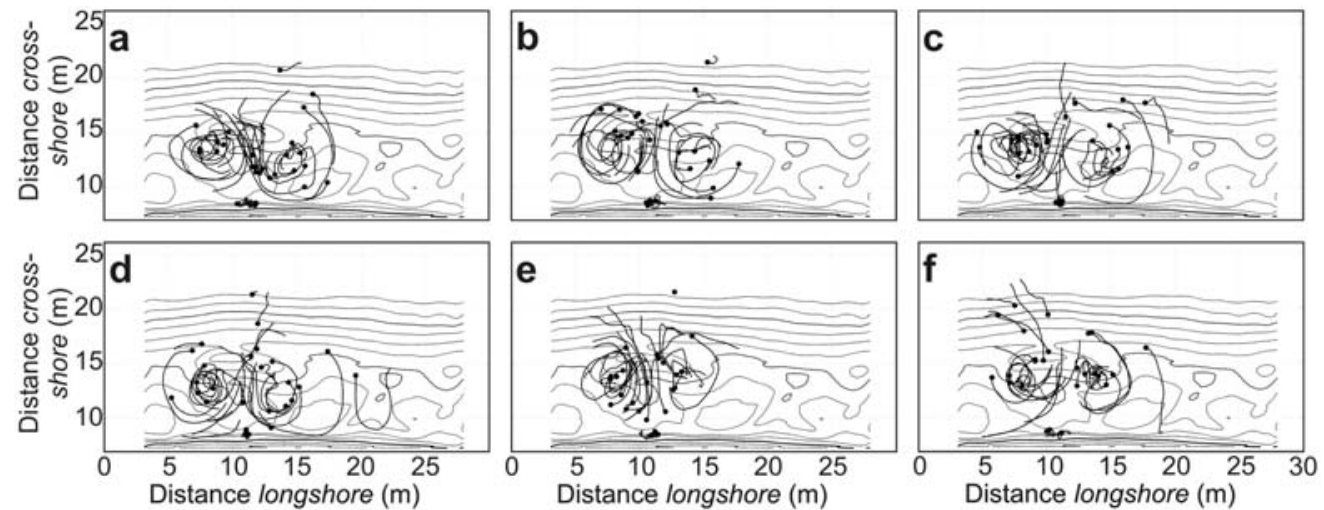

Figure 5. Trajectoires des drifters pour la morphologie en figure $2 d$ toutes les $120 \mathrm{~s}$ en partant à (a) $t=420 \mathrm{~s}$. Les points noirs correspondent au point de départ de suivi des drifters toutes les $120 \mathrm{~s}$.

Tableau 1 Pour chaque morphologie en figure 2, récapitulatif des données le nombre de drifter entrant dans le courant d'arrachement (Ne), le nombre d'expulsés (Ns) et le taux de rétention résultant (Tr).

\begin{tabular}{lllllllll}
\hline Morphologie & $a$ & $b$ & $c$ & $d$ & $e$ & $f$ & $g$ & $h$ \\
\hline Ne & 47 & 74 & 79 & 329 & 135 & 283 & 218 & 40 \\
Ns & 12 & 22 & 35 & 27 & 16 & 30 & 10 & 17 \\
$\operatorname{Tr}(\%)$ & 74.5 & 70.3 & 55.7 & 91.8 & 88.1 & 89.4 & 95.5 & 57.5 \\
\hline
\end{tabular}

\section{Discussion et conclusions}

Nous avons décrit une base de données unique sur les circulations horizontales induites par les vagues en domaine littoral. Celle-ci, complétée par les évolutions morphodynamiques, pourra à terme servir de référence pour la calibration et la validation des modèles numériques hydro-sédimentaires. Nous avons montré que les courants d'arrachement peuvent être significativement asymétriques même pour des houles frontales et que, pour une condition de forçage donnée, l'intensité du courant d'arrachement augmente linéairement avec la tridimensionnalité de plage. En outre, les courants d'arrachement les plus intenses ont été mesurés pour des morphologies de type RBB alors que dans la modélisation conceptuelle de WRIGHT \& SHORT (1984) les courants d'arrachement sont supposés être les plus intenses pour les morphologies de type TBR. Cela se justifie aussi par le fait que, jusqu'à présent, aucune mesure eulérienne ou lagrangienne de courant d'arrachement au-dessus d'un système RBB n'avait été réalisée, ni sur le terrain ni en laboratoire. 
Des résultats importants découlent du calcul des taux de rétention : les échanges hydrosédimentaires entre les franges littorales et le plateau, ou la dangerosité d'un courant d'arrachement, ne dépendent pas seulement des conditions de houle et de la tridimensionnalité de la plage, mais aussi de la géométrie des barres sableuses qui contrôle l'asymétrie des courants d'arrachement. Les données de cette expérience sont encore en cours d'analyse, et d'autres résultats préliminaires laissent présager de nouvelles avancées scientifiques.

\section{Remerciements}

Cette étude a été réalisée dans le cadre du projet MODLIT (RELIEFS/INSU-DGA) avec un financement supplémentaire de COPTER (ANR BLANC 2005-2008) pour la réalisation de l'expérimentation physique en bassin. Les auteurs tiennent à remercier la SOGREAH pour leur support technique et les discussions autour de ces expériences, ainsi que l'assistance de G. Excoffier et L. Marcellin.

\section{Références bibliographiques}

BRUNEAU N., CASTELlE B., BONNETON P., PEDREROS R., ALMAR R., BONNETON N., BRETEL P., PARISOT J.-P., SENECHAL N. (2009). Field observations of an evolving rip current on a meso-macrotidal well-developed inner bar and rip morphology. Continental Shelf Research, 29, pp 1650-1662. doi:10.1016/j.csr.2009.05.005

FEDDERSEN F., GUZA R. T. (2003). Observation of nearshore circulation: alongshore uniformity. Journal of Geophysical Research, 108, C1. doi:10.1029/2001JC001293

HALLER M., DALRYMPLE R. (2001). Rip current instabilities. Journal of Fluid Mechanics, 433, pp 161-192.

KENNEDY A.B., THOMAS D. (2004). Drifter measurements in a laboratory rip current. Journal of Geophysical Research, 109, C08005. doi:10.1029/2003JC001927

MACMAHAN J.H., THORNTON E.B., RENIERS A.J.H.M. (2006). Rip current review. Coastal Engineering, 53, pp 191-208. doi:10.1016/j.coastaleng.2005.10.009

MACMAHAN J. H., BROWNE J., BROWNE J., THORNTON E. B., RENIERS A. J. H. M., STANTON T., HENRIQUEZ M., GALlAGHER E., MORISON J., AUSTIN M. J., SCOTT T. M., SENECHAL N. (2010). Mean lagrangian flow behavior on an open coast rip-channeled beach: a new perspective. Marine Geology, 268, pp 1-15. doi:10.1016/j.margeo.2009.09.011

Michallet H., CASTElle B., BOUCHETTE F., LAMBERT A., BERNI C., BARTHELEMY E., BONNETON P., SOUS D. (2010). Modélisation physique de la morphodynamique d'une plage barrée tridimensionnelle, $\mathrm{XI}^{\mathrm{èmes}}$ JNGCGC, Les Sables d'Olonne, pp 379-386. doi:10.5150/jngcgc.2010.045-M

WRIGHT L.D., SHORT A.D. (1984). Morphodynamic variability of surf zones and beaches: a synthesis. Mar. Geol., 56, pp 93-118. doi:10.1016/0025-3227(84)90008-2 